\title{
A Media Literacy Education Approach to High School Sexual Health Education: Immediate Effects of Media Aware on Adolescents' Media, Sexual Health, and Communication Outcomes
}

\author{
Tracy M. Scull $\mathbb{1}^{1} \cdot$ Christina V. Dodson $\mathbb{1}^{1} \cdot$ Jacob G. Geller ${ }^{1} \cdot$ Liz C. Reeder ${ }^{1} \cdot$ Kathryn N. Stump ${ }^{1}$
}

Received: 10 September 2021 / Accepted: 20 December 2021 / Published online: 3 February 2022

(c) The Author(s), under exclusive licence to Springer Science+Business Media, LLC, part of Springer Nature 2022

\begin{abstract}
Media may function as sex educators for adolescents; unfortunately, media messages often glamorize risky sexual behaviors and unhealthy relationships and neglect sexual health behaviors and communication. Media Aware is a web-based comprehensive sexual health program for high school students that uses a media literacy education approach. It is designed to improve adolescents' critical thinking about media messages and provide medically-accurate information and skills building related to sexual health and communication. A randomized controlled trial was conducted in 2019-2020 with students (grades 9 and 10;n=590) from 17 high schools across the United States. The sample was 53\% female, 58\% white/ Caucasian; and 13\% Hispanic/Latinx. One high school teacher per school and all of their 9th and 10th grade students were randomly assigned to either the intervention or delayed-intervention (control) condition. The study assessed the immediate (posttest) and short-term (3-month) effects of Media Aware on adolescents' media, sexual health, and communication outcomes. For 9 of the 17 schools, students were home from school due to the beginning of the COVID-19 pandemic during the time of their 3-month data collection, which left the short-term analyses underpowered. However, several impacts of the program were found in the immediate posttest analyses. Media Aware was found to improve sexual health knowledge and redress inaccurate normative beliefs about the frequency of risky teen sex. Media Aware also improved critical thinking about media messages with demonstrated improvements in media message deconstruction skills and decreases in the perceived realism of media messages. Moderator analyses found some differential immediate effects of the program attributable to gender. Media Aware reduced girls' normative beliefs about teen sex, generally, and increased their sexual health communication with parents as well as reduced boys' acceptance of dating violence. Students gave positive feedback about Media Aware, especially related to the online format of the program. The results from this study provide evidence that Media Aware is an effective web-based program for positively enhancing high school students' media, sexual health, and sexual health communication outcomes.
\end{abstract}

Keywords Adolescents $\cdot$ High School $\cdot$ Media Literacy Education $\cdot$ Sexual Health $\cdot$ Relationships $\cdot$ Sexual Health Communication

\section{Introduction}

Comprehensive sexual health education for adolescents has been around for decades and has been shown to be an effective method for improving adolescent sexual health

Tracy M. Scull

tscull@irtinc.us

1 Innovation Research \& Training, 5316 Highgate Drive, Suite 125, Durham, NC 27713, USA related to preventing unintended pregnancy and disease transmission (Kirby \& Laris, 2009). However, many programs focus too narrowly, and offer limited, if any, content on topics such as healthy/unhealthy relationships, gender role norms, communication skills, or sexual violence, which are key components of sexual health (Haberland and Rogow, 2015). In addition, teens are immersed in a media world. Research supports the idea that sexual media can act as a "super peer" for adolescents (Brown, Halpern, \& L'Engle, 2005), influencing sexual attitudes and behaviors (Coyne et al., 2019). Media literacy education offers a promising approach to enhancing 
sexual health education to be more effective for this generation. Media Aware is a web-based comprehensive sexual health education program for high school students that is designed to build critical analysis skills related to sexual and/or romantic media messages, sexual health knowledge, and communication skills. A small, feasibility study of the program was previously conducted within one large high school and found several positive immediate impacts of the program on adolescents' mediaand health-related outcomes (Scull, Malik, Morrison, \& Keefe, 2021). The current study seeks to extend the promising findings from the feasibility study by evaluating the program's effects with a larger and more diverse sample of high school students and aiming to examine whether effects persist, emerge, or degrade over time.

\section{Comprehensive Sexual Health Education Content}

Comprehensive sexual health education programs are essential in that they provide students with medicallyaccurate information about reproductive health. Studies show that many adolescents are not protecting themselves during sexual activity, which can have immediate and longterm impacts on their health. More than $45 \%$ of sexually active high schoolers in the US did not use a condom at last sexual contact, a factor that contributes to both the relatively high U.S. teen birth rates and the prevalence of sexually transmitted infection (STI) cases among adolescents and young adults (Kann et al., 2018). Sexual violence is a significant concern during adolescence; in one study about 27 percent of girls and about 5 percent of boys reported experiencing sexual assault or abuse by the time they were 17 (Finkelhor, Shattuck, Turner, \& Hamby, 2014). Within relationships, adolescents are also at risk of dating violence and sexual abuse from their partners. Among students who had dated in the prior year, about $7 \%$ reported experiencing sexual dating violence and $8 \%$ physical dating violence, and these percentages are higher for females (Kann et al., 2018). A 30-year review of comprehensive school-based sex education evaluations focusing on program effects beyond pregnancy and disease prevention provides evidence for the effectiveness of programs that take an inclusive, affirming, and broad approach to sex education to address topics such as gender, dating and interpersonal violence, and healthy relationships (Goldfarb \& Lieberman, 2021). Additionally, effective sexual health communication with partners (Widman, Noar, Choukas-Bradley, \& Francis, 2014) and parents (Crosby, Hanson, \& Rager, 2009) have been shown to significantly reduce risk behavior and improve sexual health. Sexual health education that incorporates these related health topics is likely to have additional health gains for adolescents.

\section{Media Influence on Adolescent Sexual Health}

Media play a prominent and important role in the lives of adolescents. Teens spend over seven hours per day using media excluding school-related media use (Rideout \& Robb, 2019). Almost half of teens say they are online "almost constantly," and virtually all have access to a smartphone (Pew Research Center, 2018). Sexual and romantic content is commonplace in media. For example, a review of television programs and films found sexual references in over $80 \%$ of each (Ward, Erickson, Lippman, \& Giaccardi, 2016). Popular music has become much more sexualized and objectifying over the past five decades (Smiler, Shewmaker, \& Hearon, 2017). Because of the many ways to conceptualize exposure to pornography (intentional, unintentional, etc.), it has been difficult to get a proper estimate of adolescent exposure. However, a review of research studies leading up to 2015 concludes that at least a sizable minority of adolescents use pornography (Peter \& Valkenburg, 2016).

Media often normalize or even glamorize sexual risk behaviors and unhealthy relationships, while omitting important sexual health behaviors. Sexual content in movies (O'Hara, Gibbons, Gerrard, Li, \& Sargent, 2012) and TV (Alexopoulos \& Taylor, 2020) is frequently devoid of the use or negotiation of contraception or protection. Unprotected sex acts have also been found to be exceedingly common in sexually explicit media (Grudzen et al., 2009). A content analysis of 50 films popular among young adults found that when sexual consent was portrayed, it was often communicated though nonverbal or implicit cues with depictions of explicit verbal sexual consent being rare (Jozkowski et al., 2019). Reality dating programs contain frequent sexual situations, often concurrent with alcohol use, but without portraying potential dangers (Kim \& Wells, 2017). Pornography videos have been found to include very high levels of physical and verbal aggression, typically enacted by males on females (Bridges, Wosnitzer, Scharrer, Sun, \& Liberman, 2010).

Meta-analyses have found exposure to sexually explicit media (Smith et al., 2016) and mainstream sexual media (Coyne et al., 2019) have significant effects on adolescent sexual attitudes and behaviors. In addition to impacting attitudes and behaviors related to adolescent sexual activity, sexual media exposure is an established contributor to the acceptance of rape myths (Johnston \& Dill-Shackleford, 2020), and research has found that exposure to sexually explicit and sexually violent media is related to more accepting attitudes towards dating and sexual violence. This suggests that sexual media exposure is also related to sexual assault victimization and perpetration, and less willingness to intervene as a bystander (Rodenhizer \& Edwards, 2019). 
Researchers have posited several theoretical frameworks to better understand the paths through which sexual media content influences adolescent sexual health. Studies have revealed that media exposure influences adolescents' normative beliefs (Coyne et al., 2019), with descriptive norms playing a specifically important role. Whereas, injunctive norms are a person's perception of the degree to important others approve of them engaging in a certain behavior (e.g., the degree to which I think my friends think that I should use a condom when I have sex), descriptive norms are perceptions of the prevalence with which other people similar to them engage in a behavior (e.g., the percentage of teens who use a condom during sex). The Integrative Model of Behavioral Prediction identifies that exposure to sexual content in entertainment media increased adolescents' sexual behaviors 12 months later by increasing descriptive norms that people like them (e.g., friends; peers) are sexually active (Bleakley, Hennessy, Fishbein, and Jordan, 2011). Exposure to sexual media content in reality television and social media posts have been found to impact adolescents' willingness to have casual sex indirectly through descriptive norms that more of their peers are engaging in casual sex (van Oosten et al., 2017). Taken together, this body of research has illuminated a need for addressing the negative influence that media can have on adolescent health.

\section{Media Literacy Education for Sexual Health Promotion}

Media literacy is commonly defined as the ability to access, analyze, evaluate, create, and act using all forms of communication (Aufderheide, 1993). Media literacy education teaches students that media messages are constructed and empowers students with the skills to actively and critically think about these messages. Scholars have proposed that media literacy skills can attenuate the potentially harmful impact of media messages, and posit that when media literate adolescents encounter a media message that promotes unhealthy behaviors, they are more likely to think critically and carefully about the messages and, in turn, less likely to be negatively influenced by it.

The impact of media on adolescent sexual health and the development of media literacy skills are important but often neglected components of comprehensive sexual health education. There is an emerging body of evidence showing that media literacy education can favorably impact attitudes and intentions toward risky health behaviors (Vahedi, Sibalis, \& Sutherland, 2018). Media literacy education has effectively addressed attitudes linking sexual activity and substance use (Rodgers, Hust, Willoughby, Wheeler, \& Li, 2019) and been used in pornography-specific applications (Rothman et al., 2018). Attitudes closely tied to sexual messages in media have also been positively impacted by media literacy education, such as body image, (Rodgers, McLean, \& Paxton, 2018) and gender role stereotypes (Liao, Chang, Lee, \& Tsai, 2020). Comprehensive media literacy education-based sexual health education has been shown to be effective at improving self-efficacy and intentions to use contraception/protection in early adolescence (Scull, Kupersmidt, Malik, \& Morgan-Lopez, 2018) and risky sexual behaviors in young adulthood (Scull, Kupersmidt, Malik, \& Keefe, 2018). However, there is a gap in media literacy education programs developed specifically for high school comprehensive sexual health promotion.

\section{The Intervention}

Media Aware is a self-paced, web-based program consisting of four lessons that can fit in four 45-min class periods. The program covers topics including gender role stereotypes, healthy and unhealthy romantic relationships, dating violence and abusive relationships, sexual assault, STI transmission, prevention, testing and treatment, pregnancy prevention, FDA-approved methods of contraception, effective sexual health communication, and informed sexual decision making. This comprehensive sexual health education program is designed to be developmentally appropriate for high school students and bridge media literacy education with medically accurate sexual health information. The development of the program was informed by dual-process theories of persuasion [e.g., Elaboration Likelihood Model (ELM; Petty \& Cacioppo, 1986); Heuristic-Systematic Model (HSM; Chaiken, Giner-Sorolla, \& Chen, 1996)], message interpretation theory (i.e., the Message Interpretation Process (MIP) model; Austin \& Johnson, 1997a), and theories of behavior change [i.e., Theories of Reasoned Action and Planned Behavior (TRA/ TPB; Ajzen, 1991; Ajzen \& Fishbein, 1975). Dual processes theories posit that messages processed through more effortful systematic processing result in longer lasting attitude change that is predictive of behavior. When systematic processing results in unfavorable thoughts being generated about a message and its contents, the message is more likely to be rejected and persuasion attenuated. Within the context of media literacy education, this suggests that equipping students with the skills and motivation to carefully and critically analyze media messages promoting risk behaviors may reduce the potentially harmful impact of these messages. The MIP model posits that, in part, logic-based perceptions of media messages (e.g., perceived similarity to and realism of media messages) predict outcome expectancies and behavioral intentions related to behaviors being portrayed in the messages. The MIP model suggests that students' who perceive media messages promoting risk 
behaviors to be realistic and similar to their own life are more likely to be impacted by these messages. There is a large body of research establishing that the constructs in the TRA/TPB, including attitudes, self-efficacy, and normative beliefs, impact behavioral intentions and, ultimately, behaviors, including those related to sexual health. Informed by these theories, the conceptual framework that guided program development posited that more deliberate processing of media messages through a critical lens can lead to more negative attitudes toward and redressed normative beliefs about risk behaviors promoted in media messages, and subsequently lower the likelihood of enacting the risk behaviors.

Media literacy education is the basis for critical analysis and deconstruction of media messages. The focus is on evaluating the accuracy and realism of the messages, identifying biases and missing information in the messages, and accessing medically-accurate health information. This program specifically targets media messages relating to sex and gender roles, relationship health, sexual violence, and communication. In addition to peer videos and animated lesson slides, students interact with the content through quizzes and simulations, which enable practice of the knowledge and skills taught in the program. The program development process used a multidisciplinary approach and incorporated feedback from experts in adolescent sexual health, media effects research, instructional design, developmental psychology, web application development, and multimedia design. A small, feasibility study of the program was previously conducted within one large high school and found several positive immediate impacts of the program on adolescent outcomes, including critical thinking about media messages, sexual health, and sexual health communication (Scull, Malik, Morrison, \& Keefe, 2021). Specifically, compared to a delayed-intervention group, Media Aware increased students' cognitive elaboration when thinking about an advertisement that used sex appeals to promote alcohol, and decreased their perceive realism of and similarity to media messages. The program increased sexual health knowledge and efficacy to use protection if they decided to have sex, decreased normative beliefs about teen sexual activity and teen risky sexual activity, and increased intentions to communicate about sexual health with parents, partners, and medical professionals. Students reported being less willing to "hook-up" if their girlfriend or boyfriend wanted to but they were not sure that they did, and males reported being less willing to have unprotected sex. The program also enhanced students' efficacy and intentions to intervene as a bystander to potential sexual assault. While the results from the feasibility study are very promising, more information is needed on how this program impacts a larger and more diverse sample of high school students as well as whether the promising impacts of the program persist, emerge, or degrade over time.

\section{Current Study}

Compared with other developmental periods, less is known about how a media literacy education program can serve as effective sexual health education for high school students and whether program impacts persist over time. The current study utilized an intent-to-treat, randomized controlled trial design with a delayed-intervention control group to evaluate the immediate (posttest) and short-term (3-month followup) efficacy of Media Aware for enhancing adolescent outcomes related to media, sexual health, and communication. The results of the feasibility study, along with theories of information and media message processing, persuasion, and health behavior change inform the current hypotheses. It is hypothesized that the program will improve adolescents' media outcomes, like critical thinking about media messages (Hypothesis 1); adolescents' sexual health outcomes, like sexual health knowledge; normative beliefs about sexual activity; and self-efficacy and intentions for bystander intervention and less risky sexual behaviors (Hypothesis 2); and adolescents' communication outcomes, like normative beliefs, self-efficacy, intentions, and behaviors for sexual health communication (Hypothesis 3). Finally, adolescents' overall evaluation of the program will be examined.

\section{Methods}

\section{Participants}

One teacher from each of 17 high schools from across the U.S. was recruited to participate with all of their 9th or 10th grade classes ( $n=17$ teachers; 39 classes; 785 students). Students were eligible to participate in the study if they were in 9th or 10th grade and were fluent in English. Students in these classrooms who were not permitted to received school-based sexual health education were not eligible to participate in the study. Twenty-one participants completed the questionnaires but were excluded from the analyses because they reported their school grade to be either 11th or 12th on the questionnaires. Overall, 590 students $(75 \%)$ participated in this study. See Table 1 for demographic characteristics of the total student sample. The sample included more girls (53\%) than boys (43\%) and $4 \%$ of the sample preferred to self-describe their gender identity. Participants identified as White (58\%); Multiracial (17\%); Black (16\%); Asian (8\%); and less than one percent American Indian/Alaska Native and Pacific Islander/Native Hawaiian. Thirteen percent of the sample reported Hispanic/ Latino ethnicity. Twenty-one percent of participants reported that they qualified for free school lunch, and 15\% 
Table 1 Sample demographic characteristics and baseline equivalence tests from pretest data collection for the total sample and by condition

\begin{tabular}{|c|c|c|c|c|c|c|c|c|c|}
\hline & \multicolumn{2}{|c|}{$\begin{array}{l}\text { Total } \\
(N=590)\end{array}$} & \multicolumn{2}{|c|}{$\begin{array}{l}\text { Intervention } \\
(N=216)\end{array}$} & \multicolumn{2}{|c|}{$\begin{array}{l}\text { Control } \\
(N=374)\end{array}$} & \multirow[b]{2}{*}{$d f$} & \multirow[b]{2}{*}{$\chi^{2}$} & \multirow[b]{2}{*}{$p$-value } \\
\hline & $n$ & $\%$ & $n$ & $\%$ & $n$ & $\%$ & & & \\
\hline Gender & & & & & & & $1^{\mathrm{a}}$ & 2.17 & 0.14 \\
\hline Male & 239 & 42.99 & 146 & 40.56 & 93 & 47.45 & & & \\
\hline Female & 293 & 52.70 & 197 & 54.72 & 96 & 48.98 & & & \\
\hline Prefer to self-describe & 24 & 4.32 & 17 & 4.72 & 7 & 3.57 & & & \\
\hline Missing & 34 & & & & & & & & \\
\hline SES (eligible for free school lunch) & & & & & & & $1^{\mathrm{a}}$ & 43.82 & $<0.0001$ \\
\hline Yes & 114 & 20.54 & 43 & 12.01 & 71 & 36.04 & & & \\
\hline No & 369 & 66.49 & 265 & 74.02 & 104 & 52.79 & & & \\
\hline Unsure & 72 & 12.97 & 50 & 13.97 & 22 & 11.17 & & & \\
\hline Missing & 35 & & & & & & & & \\
\hline Ethnicity & & & & & & & 1 & 0.20 & 0.65 \\
\hline Hispanic/Latino & 72 & 13.04 & 45 & 12.57 & 27 & 13.92 & & & \\
\hline Not Hispanic/Latino & 480 & 86.96 & 313 & 87.43 & 167 & 86.08 & & & \\
\hline Missing & 38 & & & & & & & & \\
\hline Race & & & & & & & $1^{\mathrm{a}}$ & 49.40 & $<0.0001$ \\
\hline American Indian & 4 & 0.74 & 1 & 0.28 & 3 & 1.59 & & & \\
\hline Asian & 43 & 7.93 & 21 & 5.95 & 22 & 11.64 & & & \\
\hline Black & 86 & 15.87 & 36 & 10.20 & 50 & 26.46 & & & \\
\hline Pacific Islander/Native Hawaiian & 1 & 0.18 & 1 & 0.28 & 0 & 0.00 & & & \\
\hline White & 314 & 57.93 & 243 & 68.84 & 71 & 37.57 & & & \\
\hline More than one race & 94 & 17.34 & 51 & 14.45 & 43 & 22.75 & & & \\
\hline Missing & 48 & & & & & & & & \\
\hline Sexual Experience & & & & & & & 1 & 5.33 & 0.02 \\
\hline Yes & 75 & 15.24 & 40 & 12.50 & 35 & 20.35 & & & \\
\hline No & 417 & 84.76 & 280 & 87.50 & 137 & 79.65 & & & \\
\hline Missing & 98 & & & & & & & & \\
\hline
\end{tabular}

${ }^{a}$ Chi-square analysis was conducted for binary category (male/female; yes/no; white/nonwhite). reported previous sexual experience. The average age of participants in the sample was 14.42 years $(S D=0.70)$.

\section{Procedures}

An Institutional Review Board approved of the methods and measures used in this study. For detailed information on study procedures, see the protocol paper for this study (Scull, Malik, Morrison, \& Keefe, 2020). Participating schools were randomized to either the intervention group ( $n=9$ teachers; 23 classes; 478 students) and control group ( $n=8$ teachers; 16 classes; 307 students). See Fig. 1 for the CONSORT flow diagram for this randomized control trial.

Teachers used a script to distribute consent and assent form packets to students in participating classrooms. Students were asked to review the forms with their parents and return signed copies indicating their choice to participate or not. Teachers received a $\$ 30$ incentive for each classroom that returned $80 \%$ of their forms signed, regardless of whether the students were permitted or assented to participate or not.

Study staff members were in the classroom while participating students completed the web-based pretest questionnaire on laptops during a regular class period. Students in the intervention classes completed Media Aware on their laptops during their next four to five classes. Students in the control classes received their typical health education content across the next four to five classes. Teachers in the control classes were asked to refrain from teaching sexual health topics until data collection was completed. Then, study staff members returned to the classroom to facilitate students in both conditions completing the web-based posttest questionnaire. Study staff members returned for a final class period approximately 3 months after the pretest questionnaire was completed to facilitate the students completing the final web-based follow-up questionnaire. At this point, teachers in the control condition could have their students complete Media Aware. 


\section{7 schools ( $N=785$ students)}

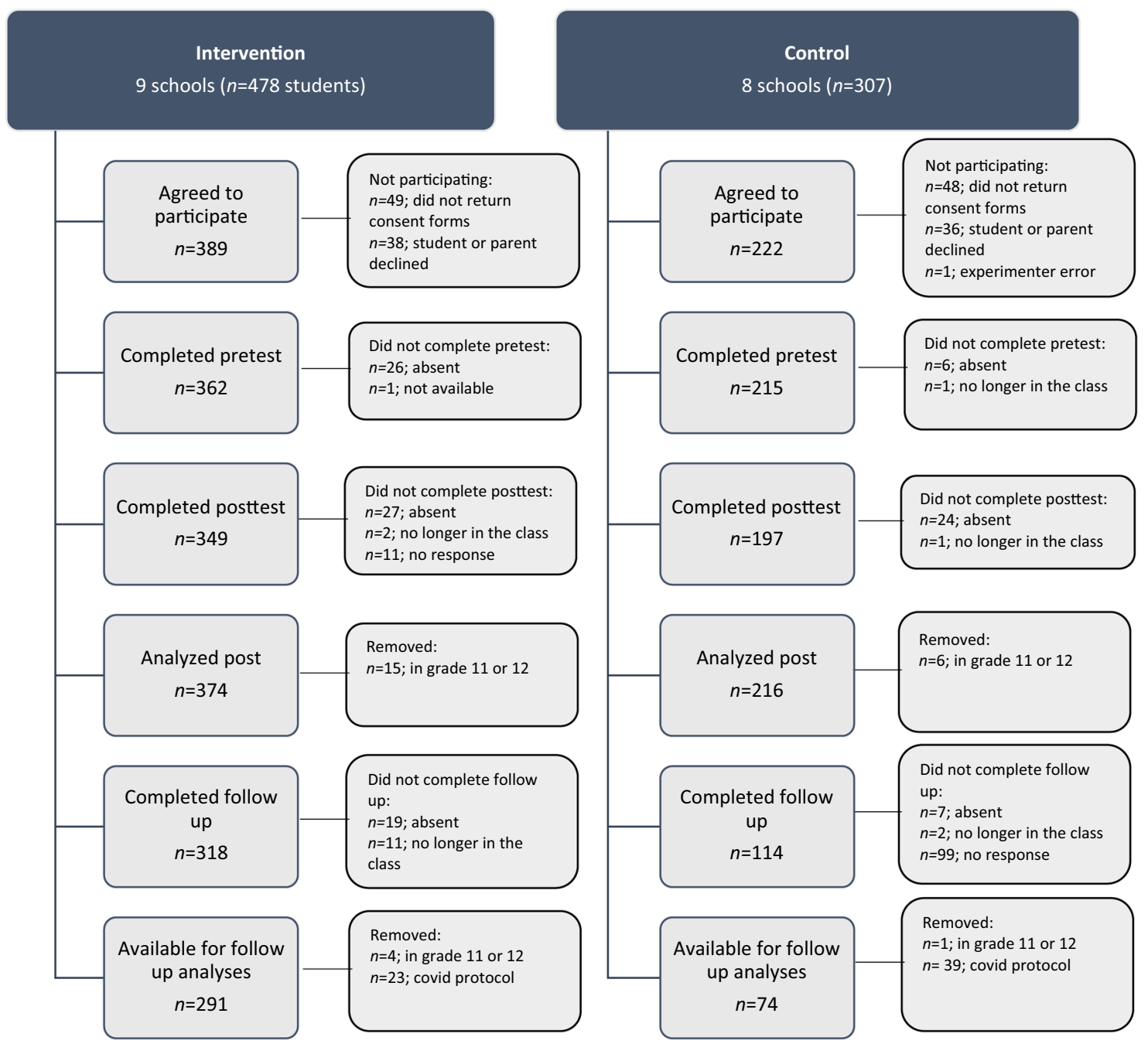

Fig. 1 CONSORT diagram

It was necessary to alter the in-person data collection protocol near the end of the study due to the COVID-19 pandemic. Up until the pandemic started in the U.S., the study team monitored students completing their web-based questionnaires by being physically present in each classroom. Once lockdowns began in Spring 2020, all participating schools sent their students home for remote learning. At this point, 16/17 schools had completed their posttest data collection, and 8/17 schools had also completed their 3-month follow-up data collection. After adjusting the protocol and receiving IRB approval, the study team attempted to collect data from the remaining students at the appropriate timepoints by emailing the students links to the web-based questionnaires.

\section{Measures}

Participants completed questionnaires at pretest, posttest, and 3-month follow-up timepoints. The questionnaires were exactly the same, except for the posttest for the intervention group, which included several program evaluation questions. Example items for scaled constructs can be found in the protocol paper (Scull, Malik, Morrison, \& Keefe, 2020).

\section{Media outcomes}

Perceived realism of media messages Participants responded to five items to assess how realistic they perceive 
media messages to be [adapted from Austin and Johnson (1997), 4-point Likert scale, $1=$ strongly disagree; $4=$ strongly agree, $\alpha=0.79]$.

Skepticism of media messages Participants responded to three items to assess their skepticism of media messages about sexual behavior and substance use [adapted from Scull, Kupersmidt, Malik, \& Keefe (2018), 4-point Likert scale, $1=$ strongly disagree $;=$ strongly agree, $\alpha=0.73$ ].

Media message deconstruction skills Participants viewed a print advertisement for an alcoholic beverage that used a sexual/romantic ad hook. To assess media message deconstruction skills, participants were asked to respond to three open-ended prompts related to the print advertisement (e.g., Tell us about this advertisement in the space below.). Their responses were qualitatively coded by two research team members who were blind to data collection timepoint and condition and using a coding rubric used in previous research (Scull, Kupersmidt, Malik, \& Keefe, 2018; Scull, Malik, Morrison, \& Keefe, 2021) that assigned points to categories (i.e., goal, product, visuals, language, target audience, implied messages, and missing information). Prior to coding, inter-coder reliability was established through training sets of similar data from another study $(\kappa>0.70)$. Reliability was checked periodically throughout coding to monitor drift. Coding discrepancies were resolved by a third (and if necessary, a fourth) trained coder. Coders could not achieve reliability for one category (i.e., target audience) because the topic was so infrequently mentioned in the responses. The six reliable category scores were summed to create the overall media message deconstruction skills composite variable, with a total potential score ranging from a low of 0 to a high of 13 . The reliability for the mean composite variable was good $(\kappa=0.81)$.

Cognitive elaboration of a media message Participants responded on a 4-point Likert scale $(1=$ not much at all, $4=a$ lot $)$ to items about their cognitive elaboration of the ad or the extent to which they spent viewing, thinking, and paying attention to the ad [3 items; adapted from Shiv, Britton, and Payne (2004); $\alpha=0.87$ ]

Counterarguing of a media message Participants responded on a 4-point Likert scale $(1=$ not much at all, $4=a$ lot $)$ to items about their counterarguing of the ad or how much they were internally criticizing or arguing against the messages in the ad [4 items; adapted from Moyer-Gusé and Nabi (2010); $\alpha=0.84]$.

Perceived completeness of a media message Participants responded on a 4-point Likert scale $(1=$ incomplete; $4=$ complete) about their perceptions of the completeness of the ad [1 item; from Scull, Malik, Keefe, \& Schoemann (2019)].
Perceived credibility of a media message Participants responded on a 4-point Likert scale $(1=$ strongly disagree; $4=$ strongly agree) to items to assess their perceptions of the credibility of the ad [ 3 items; [adapted from MacKenzie, Lutz, and Belch (1986); $\alpha=0.80]$.

\section{Sexual health outcomes}

Acceptance of strict gender role stereotypes, dating violence, and rape myths Participants responded to a series of statements with a 4-point Likert scales $(1=$ strongly disagree; $2=$ disagree $; 3=$ agree $; 4=$ strongly agree). The items assessed the acceptance of strict gender role stereotypes [6 items; adapted from Foshee et al. (2005); $\alpha=0.80$ ], acceptance of dating violence [ 3 items; adapted from Foshee et al. (2005); $\alpha=0.71$, and acceptance of rape myths [5 items; adapted from McMahon and Farmer (2011); $\alpha=0.87]$.

Efficacy for bystander intervention Participants were asked about their efficacy for bystander intervention in the presence of a potential sexual assault [5 items; $(0=$ can't do, $100=$ very certain $)$; adapted from Banyard, Plante, and Moynihan (2005); $\alpha=0.84]$

Intentions for bystander intervention Participants were asked about their intentions for bystander intervention [4 items; 4-point Likert scale ( $1=$ not at all likely, $4=$ extremely likely); adapted from Banyard et al. (2005); $\alpha=0.81]$.

Sexual health knowledge Participants completed an inventory of objective sexual health knowledge questions, including multiple choice, true/false, and multi-response questions (8 items; range 0-13 correct answers).

Teen sex and risky teen sex descriptive norms To assess descriptive normative beliefs, Participants were asked to rate how many teens engage in certain behaviors (rated from $0 \%$ to $100 \%$ ), including teen sex (5 items; $\alpha=0.85$ ) and risky teen sex ( 3 items; $\alpha=0.85$ ). The descriptive normative belief measures were adapted from Scull, Kupersmidt, Malik, \& Keefe (2018).

Intent to have sex Participants were asked how likely it is that they will have any type of sexual contact with another person (including oral, anal, and vaginal sex and genital-to-genital contact) in the next year [1-item; 4-point Likert scale $(1=$ not at all likely, $4=$ extremely likely); adapted from L'Engle, Brown, and Kenneavy (2006)].

Willingness to hook up with a boyfriend/girlfriend Participants were given a vignette and asked to rate how willing 
they would be to hook up if their girlfriend or boyfriend wanted to, but they were not sure that they wanted to $[1=$ very unwilling, $4=$ very willing; adapted from Gibbons, Gerrard, Blanton, and Russell (1998)].

Willingness to have sex with a boyfriend/girlfriend Participants were given a vignette and asked to rate how willing they would be to have sex if their girlfriend or boyfriend says "I love you" and wants to have sex $[1=$ very unwilling, 4 = very willing; adapted from Gibbons, Gerrard, Blanton, and Russell (1998)].

Willingness for unprotected sex with a boyfriend/girlfriend Participants were given a vignette and asked to rate how willing they would be to have sex with a boyfriend or girlfriend without protection $[1=$ very unwilling, $4=$ very willing; adapted from Gibbons, Gerrard, Blanton, and Russell (1998)].

Contraception/protectiondescriptive norms To assess descriptive normative beliefs, Participants were asked to rate how many teens use contraception/protection during sex (rated from 0\% to 100\%; (3 items; $\alpha=0.79$ ). The descriptive normative belief measure was adapted from Scull, Kupersmidt, Malik, \& Keefe (2018).

Efficacy for using contraception/protection Participants rated their efficacy to use contraception/protection [3 items; 4-point Likert scale $(1=$ strongly disagree, $4=$ strongly agree); adapted from Soet, Dudley, and Dilorio (1999). $\alpha=$ $0.68]$;

Intention to use contraception/protection Participants rated their intention to use a condom for vaginal or anal sex; other birth control for vaginal sex; and protection (i.e., a condom or dental dam) for oral sex [3 separate items; 4-point Likert scale $(1=$ not at all likely, $4=$ extremely likely $)$; adapted from Jemmott and Jemmott (1991)].

\section{Sexual behaviors and contraception/protection behaviors}

Participants reported sexual behaviors, including if they had oral, vaginal, or anal sex, and if so, did they use a barrier method the last time ( 6 items; $1=y e s, 2=n o$, 3 = unsure).

\section{Communication outcomes}

Sexual health communication descriptive norms To assess descriptive normative beliefs, participants were asked to rate how many teens their age communicated with partners, parents, and medical professionals about sexual health [3 items; 0-100; (Scull, Kupersmidt, Malik, \& Keefe, 2018); $\alpha=0.82]$.
Efficacy to communicate about sexual health Participants were also asked to rate their efficacy to communicate with partners, parents, and medical professional about sexual health [ 3 items; 4-point Likert scale $(1=$ strongly disagree, 4 strongly agree); adapted from Scull, Malik, \& Kupersmidt (2014); $\alpha=0.74]$

Efficacy to negotiate contraception/protection use Participants were also asked to rate their efficacy for negotiating using protection/contraception with a partner, if they decide to have sex $[2$ items; 4-point Likert response scale $(1=$ strongly disagree, 4 strongly agree); adapted from Soet et al. (1999); $r=0.81]$.

Intent to communicate about sexual health Participants reported their intentions to communicate with partners, parents, and medical professionals prior to sexual behavior [3 items; 4-point Likert scale $(1=$ not at all likely; $4=$ extremely; $\alpha=$ 0.75); adapted from Scull, Malik, \& Kupersmidt (2014)].

Sexual health communication behaviors Participants reported their frequency of sexual health communication with partners, parents, and medical professionals [ 3 separate items; 4-point Likert scale $(1=$ never, $4=$ often $)$; adapted from Scull, Malik, \& Kupersmidt (2014)].

\section{Background demographic measures}

At pretest only, participants reported their age, grade level, race and ethnicity, sex, gender identity, SES (i.e., eligibility for free or reduced lunch), and school grades (e.g., "Mostly As," "Some As and Bs," "Mostly Bs", etc.).

\section{Program evaluation measures}

At posttest only, participants in the intervention group responded to items related to their experiences completing the program using a 4 -point Likert scale $(1=$ not very to $4=$ very $)$. They were asked 1) how interesting the program was, 2) how much they learned from the program, and 3) how glad they were to have used the program. Also, they noted their agreement to the following statements using a 4-point Likert scale $(1=$ strongly disagree to $4=$ strongly agree $): 1)$ I liked that I could do this program on a computer; 2) I liked this program better than a teacher teaching sexual health education in the classroom; 3) I felt less embarrassed taking this program than having a teacher reaching sexual health; and 4) this is a good program for teens to learn about sexual health.

\section{Process measures}

The Learning Management System (LMS) that housed the web-based program captured information on students' use 
of the program (percent of lessons completed out of 10) and their scores on the 10 section quizzes (percent correct).

\section{Statistical Analysis}

A power analysis was conducted for the RCT. Based upon power $(0.80)$, effect size $(d=0.40)$, estimated average cluster size of students per school (40), and ICC for school effects (0.05), the results revealed that 17 schools would be needed to participate in the study (Optimal Design Software; Raudenbush et al., 2011). All 17 schools completed their pretest data collection prior to the COVID-19 pandemic. All but one school completed their posttest data collection prior to the pandemic. However, only 8 of the 17 schools completed their follow-up data collection prior to the pandemic. During the pandemic, email links to the posttest and the follow-up were sent to students at the appropriate time. However, very few students completed these questionnaires. The posttest analyses were conducted both with and without data from the one school that completed their posttest data collection during the COVID pandemic and no change was seen in the results; thus, the posttest results that are reported in this manuscript include data from all 17 schools. However, the planned follow-up analyses are not reported. The follow-up questionnaire completion rates for schools during the pandemic were markedly lower than those schools that finished the study prior to the pandemic. In addition, there are concerns about potential differences in students' responses that may have been influenced by longer periods of social distancing and quarantining (i.e., romantic relationships). Unfortunately, excluding data from schools that completed the follow-up questionnaire during the pandemic leaves the follow-up analyses underpowered.

Prior to data collection, for the sake of parsimony, two edits were made to the analysis plan previously published in the study protocol paper (Scull, Malik, Morrison, \& Keefe, 2020): 1) perceived similarity was dropped from the outcome analyses and 2) intentions to have sexual activity was reduced from a four-item scale to the most proximal item (i.e., in the next year). In response to psychometric analyses and prior to the outcome analyses, three other variables were revised. Two scaled outcome variables were shortened to improve the scale's reliability (i.e., dating violence norms; media skepticism). In addition, the items related to intent to use contraception/protection were used separately, rather than as a scale, and one item was eliminated from the efficacy for contraception/protection scale that was only relevant for participants with ovaries (i.e., If I wanted to, I could get birth control other than condoms, such as birth control pills, IUD, or injection). Finally, two longer-term behavioral outcomes that were proposed to be studied in the follow-up analyses were dropped from the analysis plan because of complications due to the COVID pandemic (i.e., sexual debut; protection use).

Chi-square analyses and t-tests were used to test for baseline equivalence between the intervention and control groups. Descriptive statistics were calculated for the outcome variables and process measures (i.e., completion; quiz scores). The intent-to-treat outcome analyses were conducted using Statistical Analysis System (SAS) statistical software package, version 9.4 (SAS Institute Inc., Cary, NC, USA). A series of multilevel regression models were estimated to determine the association between condition and each outcome, while controlling for the conditional non-independence of observations (i.e., student nested within teacher). Fixed effects included condition (intervention vs. control), the adolescent's pretest outcome score, and covariates (age, SES, race, sexual experience). The models included a random intercept for teacher and degrees of freedom were adjusted using the Satterthwaite adjustment. In addition, the analyses tested gender as a moderator of the effectiveness of the program. Results of the outcome analyses are reported as adjusted posttest mean scores. Finally, descriptive statistics were calculated for the quantitative program feedback data.

Due to high rates of missing data in some of the background covariate variables, all data were multiply imputed 100 times to accurately estimate and recover the missing values. The imputation procedure was stratified by condition to maintain any group differences resulting from the intervention. All multilevel analysis models were conducted on each of the 100 imputed datasets and all results were pooled using Rubin's Rules, to adjust for between- and within-imputation variance of estimates (Rubin, 1987).

\section{Results}

\section{Preliminary Analyses}

See Table 1 for the results of chi-square analyses for the testing of baseline equivalence in sample demographic characteristics between the intervention and control groups. The results revealed significant differences $(p<0.05)$ in the two groups with respect to race (white/nonwhite), SES (free lunch/no free lunch), and sexual experience (yes/no). The two groups did not differ significantly with respect to gender (male/female) or ethnicity. A t-test revealed that participants in the intervention group were slightly younger $(M=14.33$, $S D=0.58)$ than participants in the control group $[M=$ $14.60, S D=0.86 ; t(253.27)=3.77, p=0.0002]$. Demographic characteristic variables that were found to be significantly different between the conditions were subsequently used as covariates in the outcome analyses. Means, standard deviations, and ranges were calculated for 
Table 2 Descriptive statistics for outcome variables at pretest for the total sample and by condition

\begin{tabular}{|c|c|c|c|c|c|c|c|c|}
\hline \multirow[b]{2}{*}{ Measure } & \multicolumn{2}{|c|}{ Intervention } & \multicolumn{2}{|c|}{ Control } & \multicolumn{2}{|l|}{ Total } & \multirow[b]{2}{*}{$\min$} & \multirow[b]{2}{*}{$\max$} \\
\hline & $M$ & $S D$ & $M$ & $S D$ & $M$ & $S D$ & & \\
\hline \multicolumn{9}{|l|}{ Media } \\
\hline Perceived realism of media messages & 2.28 & 0.53 & 2.22 & 0.57 & 2.27 & 0.55 & 1.00 & 4.00 \\
\hline Skepticism of media messages & 2.89 & 0.57 & 2.80 & 0.64 & 2.26 & 0.55 & 1.00 & 4.00 \\
\hline Media message deconstruction skills & 5.97 & 1.91 & 5.33 & 2.02 & 5.73 & 1.97 & 0.00 & 12.00 \\
\hline Cognitive elaboration of a media message & 2.13 & 0.67 & 2.01 & 0.69 & 2.08 & 0.68 & 1.00 & 4.00 \\
\hline Counterarguing of a media message & 2.00 & 0.75 & 1.89 & 0.69 & 1.96 & 0.73 & 1.00 & 4.00 \\
\hline Perceived completeness of a media message & 2.24 & 0.88 & 2.29 & 0.89 & 2.26 & 0.88 & 1.00 & 4.00 \\
\hline Perceived credibility of a media message & 2.22 & 0.68 & 2.12 & 0.73 & 2.19 & 0.70 & 1.00 & 4.00 \\
\hline \multicolumn{9}{|l|}{ Sexual health } \\
\hline Acceptance of strict gender role stereotypes & 1.42 & 0.44 & 1.53 & 0.48 & 1.47 & 0.46 & 1.00 & 3.50 \\
\hline Acceptance of dating violence & 1.43 & 0.48 & 1.50 & 0.54 & 1.46 & 0.50 & 1.00 & 4.00 \\
\hline Acceptance of rape myths & 1.32 & 0.49 & 1.45 & 0.58 & 1.38 & 0.53 & 1.00 & 4.00 \\
\hline Efficacy for bystander intervention & 74.80 & 20.20 & 74.92 & 21.41 & 74.79 & 20.64 & 0.00 & 4.00 \\
\hline Intent for bystander intervention & 3.19 & 0.55 & 3.09 & 0.56 & 3.15 & 0.56 & 1.00 & 4.00 \\
\hline Sexual health knowledge & 10.87 & 1.62 & 10.57 & 1.64 & 10.72 & 1.63 & 4.00 & 13.00 \\
\hline Teen sex descriptive norms & 41.96 & 19.15 & 43.49 & 22.18 & 42.80 & 20.28 & 0.00 & 100.00 \\
\hline Risky teen sex descriptive norms & 37.22 & 20.59 & 40.68 & 24.62 & 38.74 & 22.13 & 0.00 & 100.00 \\
\hline Intent to have sex & 1.78 & 0.93 & 1.90 & 1.03 & 1.83 & 0.97 & 1.00 & 4.00 \\
\hline Willingness to hook-up with a bfriend/gfriend & 2.05 & 0.79 & 2.14 & 0.81 & 2.08 & 0.80 & 1.00 & 4.00 \\
\hline Willingness to have sex with a bfriend/gfriend & 2.28 & 0.96 & 2.32 & 0.96 & 2.29 & 0.96 & 1.00 & 4.00 \\
\hline $\begin{array}{l}\text { Willingness to have unprotected sex with a } \\
\text { bfriend/gfriend }\end{array}$ & 1.53 & 0.73 & 1.70 & 0.85 & 1.59 & 0.78 & 1.00 & 4.00 \\
\hline Contraception/protection descriptive norms & 53.46 & 20.26 & 53.17 & 20.75 & 53.21 & 20.41 & 0.00 & 100.00 \\
\hline Efficacy for using contraception/protection & 3.04 & 0.70 & 3.01 & 0.68 & 3.03 & 0.70 & 1.00 & 100.00 \\
\hline Intent to use condom for vaginal or anal sex & 3.63 & 0.64 & 3.51 & 0.78 & 3.58 & 0.69 & 1.00 & 4.00 \\
\hline Intent to use other birth control for vaginal sex & 2.90 & 0.88 & 2.84 & 0.95 & 2.88 & 0.91 & 1.00 & 4.00 \\
\hline Intent to use protection for oral sex & 2.95 & 0.98 & 2.79 & 1.05 & 2.89 & 1.01 & 1.00 & 4.00 \\
\hline \multicolumn{9}{|l|}{ Communication } \\
\hline $\begin{array}{l}\text { Sexual health communication } \\
\text { descriptive norms }\end{array}$ & 34.07 & 20.04 & 36.72 & 21.32 & 34.97 & 20.51 & 0.00 & 91.67 \\
\hline Efficacy to communication about sexual health & 2.86 & 0.61 & 2.89 & 0.69 & 2.87 & 0.64 & 1.00 & 4.00 \\
\hline $\begin{array}{l}\text { Efficacy to negotiate contraception/ } \\
\text { protection use }\end{array}$ & 3.32 & 0.61 & 3.25 & 0.66 & 3.29 & 0.63 & 1.00 & 4.00 \\
\hline Intent to communication about sexual health & 2.70 & 0.69 & 2.73 & 0.74 & 2.71 & 0.71 & 1.00 & 4.00 \\
\hline Sexual health communication with parent & 2.01 & 0.96 & 1.89 & 0.88 & 1.97 & 0.93 & 1.00 & 4.00 \\
\hline $\begin{array}{l}\text { Sexual health communication with bfriend/ } \\
\text { gfriend }\end{array}$ & 1.80 & 1.03 & 1.87 & 1.05 & 1.82 & 1.03 & 1.00 & 4.00 \\
\hline $\begin{array}{l}\text { Sexual health communication with med } \\
\text { professional }\end{array}$ & 1.90 & 0.87 & 1.83 & 0.89 & 1.88 & 0.88 & 1.00 & 4.00 \\
\hline
\end{tabular}

the outcome variables for the total sample and separately by condition (see Table 2).

Descriptive statistics on the intervention group's use of Media Aware were also calculated. Three hundred eightysix students received access to the program. Twelve students $(3.10 \%)$ never began the program. The 374 students that did begin the program completed an average of $88.26 \%$ of the ten sections $(S D=25.31 \%)$ and scored an average of $79.57 \%$ correct $(S D=21.11 \%)$ on the section quizzes.

\section{Main Outcome Analyses}

Analyses were conducted to test the hypotheses that the program would positively impact adolescents' media, sexual health, and communication outcomes. Detailed information on the intent-to-treat outcome analyses for posttest effects can be found in Table 3. First, the analyses revealed two immediate main effects of the program on adolescent media outcomes. At posttest, students who received Media 
Table 3 Parameter estimates, adjusted least squares means, standard errors, $p$ values, and effect sizes (Cohen's $d$ ) for the outcome analyses at posttest

\begin{tabular}{|c|c|c|c|c|c|c|c|c|c|}
\hline & \multirow[b]{2}{*}{ Outcome } & \multirow[b]{2}{*}{$b$} & \multirow[b]{2}{*}{$S E$} & \multicolumn{2}{|c|}{ Intervention } & \multicolumn{2}{|c|}{ Control } & \multirow[b]{2}{*}{$P$} & \multirow[b]{2}{*}{$d$} \\
\hline & & & & $M$ & $S E$ & $M$ & $S E$ & & \\
\hline \multirow[t]{7}{*}{ Media } & Perceived realism of media messages & -0.15 & 0.07 & 2.00 & 0.04 & 2.15 & 0.05 & 0.02 & 0.23 \\
\hline & Skepticism of media messages & 0.13 & 0.09 & 2.99 & 0.06 & 2.86 & 0.07 & 0.16 & \\
\hline & Media message deconstruction skills & 1.28 & 0.65 & 4.66 & 0.45 & 3.38 & 0.46 & 0.05 & 0.17 \\
\hline & Cognitive elaboration of a media message & 0.12 & 0.07 & 1.97 & 0.04 & 1.85 & 0.05 & 0.07 & \\
\hline & Counterarguing of a media message & 0.12 & 0.08 & 2.01 & 0.05 & 1.89 & 0.06 & 0.15 & \\
\hline & Perceived completeness of a media message & -0.07 & 0.11 & 2.10 & 0.06 & 2.17 & 0.09 & 0.53 & \\
\hline & Perceived credibility of a media message & -0.08 & 0.07 & 2.04 & 0.04 & 2.12 & 0.05 & 0.23 & \\
\hline \multirow[t]{17}{*}{ Sexual Health } & Acceptance of strict gender role stereotypes & -0.02 & 0.06 & 1.51 & 0.03 & 1.54 & 0.04 & 0.70 & \\
\hline & Acceptance of dating violence & -0.07 & 0.06 & 1.41 & 0.04 & 1.48 & 0.05 & 0.27 & \\
\hline & Acceptance of rape myths & 0.01 & 0.05 & 1.36 & 0.03 & 1.35 & 0.04 & 0.91 & \\
\hline & Efficacy for bystander intervention & 1.41 & 1.95 & 74.90 & 1.13 & 73.49 & 1.52 & 0.47 & \\
\hline & Intent for bystander intervention & 0.02 & 0.08 & 3.05 & 0.05 & 3.03 & 0.06 & 0.80 & \\
\hline & Sexual health knowledge & 0.50 & 0.20 & 11.24 & 0.12 & 10.74 & 0.16 & 0.01 & 0.26 \\
\hline & Teen sex descriptive norms & -4.61 & 2.60 & 33.59 & 1.54 & 38.20 & 2.00 & 0.08 & \\
\hline & Risky teen sex descriptive norms & -5.23 & 2.74 & 31.47 & 1.59 & 36.70 & 2.11 & 0.05 & 0.19 \\
\hline & Intent to have sex & 0.19 & 0.10 & 2.01 & 0.06 & 1.81 & 0.08 & 0.06 & \\
\hline & Willingness to hook up with bfriend/gfriend & 0.06 & 0.07 & 1.95 & 0.04 & 1.89 & 0.06 & 0.41 & \\
\hline & Willingness to have sex with bfriend/gfriend & -0.03 & 0.09 & 2.25 & 0.05 & 2.29 & 0.07 & 0.70 & \\
\hline & Willingness for unprotected sex with bfriend/gfriend & -0.02 & 0.09 & 1.62 & 0.06 & 1.64 & 0.07 & 0.84 & \\
\hline & Contraception/protection descriptive norms & -3.21 & 2.66 & 51.28 & 1.61 & 54.50 & 2.08 & 0.23 & \\
\hline & Efficacy for using contraception/protection & 0.03 & 0.09 & 3.03 & 0.05 & 3.00 & 0.07 & 0.76 & \\
\hline & Intent to use condom for vaginal or anal sex & 0.03 & 0.13 & 3.39 & 0.08 & 3.36 & 0.10 & 0.82 & \\
\hline & Intent to use other birth control for vaginal sex & 0.12 & 0.14 & 2.89 & 0.08 & 2.78 & 0.10 & 0.42 & \\
\hline & Intent to use protection for oral sex & 0.18 & 0.14 & 2.90 & 0.08 & 2.72 & 0.11 & 0.13 & \\
\hline \multirow[t]{7}{*}{ Communication } & Sexual health communication descriptive norms & 2.71 & 2.54 & 39.35 & 1.49 & 36.64 & 1.94 & 0.29 & \\
\hline & Efficacy to communicate about sexual health & 0.08 & 0.10 & 2.87 & 0.07 & 2.79 & 0.07 & 0.44 & \\
\hline & Efficacy to negotiate contraception/protection use & 0.06 & 0.10 & 3.20 & 0.06 & 3.14 & 0.08 & 0.55 & \\
\hline & Intent to communicate about sexual health & 0.07 & 0.10 & 2.73 & 0.07 & 2.66 & 0.07 & 0.47 & \\
\hline & Sexual health communication with parent & 0.20 & 0.12 & 2.11 & 0.07 & 1.91 & 0.10 & 0.10 & \\
\hline & Sexual health communication with bfriend/gfriend & 0.16 & 0.12 & 2.00 & 0.07 & 1.84 & 0.09 & 0.18 & \\
\hline & Sexual health communication with med professional & 0.00 & 0.12 & 1.98 & 0.07 & 1.99 & 0.09 & 0.97 & \\
\hline
\end{tabular}

Positive effect sizes reflect differences in the expected direction.

Aware reported lower perceived realism of media messages and more advanced media deconstruction skills, compared to students in the control group. No other immediate main effects of the program on adolescent media outcomes were found. Next, the analyses revealed two immediate main effects of the program on adolescent sexual health outcomes. At posttest, compared with students in the control group, students who received Media Aware reported lower descriptive normative beliefs about risky teen sex and scored more correct answers on the sexual health knowledge questions. No other immediate main effects of the program on adolescent sexual health outcomes were found. Finally, the analyses did not reveal any immediate main effects of the program on adolescent communication outcomes.

\section{Gender Moderator Analyses}

Intent-to-treat analyses examined the interaction of gender and condition at posttest. There was a significant interaction of gender and condition for acceptance of dating violence $(b=-0.19, p<0.05)$ At posttest, boys in the intervention group reported less acceptance of dating violence $(M=$ $1.44, S D=0.05)$ than boys in the control group $(M=1.60$, $S D=0.07 ; d=0.29)$. The same effect was not present among girls $(d=-0.06)$. There was a significant interaction 
of gender and condition for descriptive normative beliefs about teen sex $(b=8.06, p<0.05)$. At posttest, girls in the intervention group reported lower estimates of sex amongst their peers $(M=32.06, S D=1.69)$ than girls in the control group $(M=40.44, S D=2.44 ; d=0.38)$ The same effect was not present among boys $(d=0.01)$. Finally, there was a significant interaction of gender and condition for sexual health communication with a parent $(b=-0.36, p<0.05)$. At posttest, girls in the intervention group reported more frequent sexual health communication with a parent $(M=$ $2.17, S D=0.08)$ than girls in the control group $(M=1.80$, $S D=0.12 ; d=0.37)$. The same effect was not present among boys $(d=0.01)$.

\section{Program Evaluation}

Most participants who used Media Aware as part of the intervention group reported that the program was at least a little interesting (73.09\%); that they learned at least a little bit (80.06\%); and that they were at least a little bit glad they used the program $(80.31 \%)$. The vast majority of participants agreed or strongly agreed that Media Aware is a good program for teens to learn about sexual health $(80.12 \%)$. Most liked that they could do the program on a computer (79.57\%) and liked this program better than a teacher teaching sexual health education in the classroom $(63.86 \%)$. Most students reported that they felt less embarrassed taking this program than having a teacher teaching sexual health $(63.12 \%)$.

\section{Discussion}

While research supports the teaching of comprehensive sexual health education for improving adolescents' health outcomes, most programs take a narrow approach by focusing primarily on the prevention of unintended pregnancy and STIs. Adolescents also need sexual health education that addresses sexual violence, relationship health, and the development of sexual health communication skills. In addition, media are sexual socialization agents for adolescents, and often present the drama associated with sex and relationships but rarely model healthy behaviors and sexual health communication. Teaching adolescents critical thinking skills that can be applied to media messages that are themed around sex and relationships can promote adolescent health. The current study extends upon prior work and aims to fill these gaps by presenting the findings from a randomized controlled trial evaluating the impact of Media Aware, a web-based, comprehensive sexual health education program for high school students that uses a media literacy education approach.

The analyses revealed that the program showed immediate improvements in adolescents' critical thinking about media messages, descriptive normative beliefs related to sexual health, boys' acceptance of dating violence, and girls' sexual health communication behaviors with parents, replicating many findings found in the feasibility study of the program. This is the first study to rigorously evaluate the efficacy of a web-based media literacy education program for comprehensive sexual health education among high school students, and these results reveal that the Media Aware program can effectively impact students' media, sexual health, and communication outcomes.

Media Aware was developed to improve adolescents' critical thinking about media messages and provide them with accurate health information that is often left out of media messages with sexual and romantic themes. Adolescents who received the program had more accurate sexual health information and were more likely to find media messages to be unrealistic. These adolescents also were found to have more advanced media deconstruction skills when processing an alcohol advertisement with a sexual/ romantic theme. Taken together, this suggests that when the Media Aware adolescents process media messages depicting unhealthy behaviors, they may be motivated to engage in systematic processing that results in more negative thoughts about the advertisement and the behaviors communicated within the message (e.g., alcohol use can help me get romantic attention). In theory, this processing would then result in rejecting the media message and attenuating its potentially harmful effects. Adolescents that apply a less critical eye to the advertisement, and that may perceive media messages as more realistic and/or know less about sexual health, are thus more likely be persuaded by the messages found in the ad and believe that alcohol use can have positive effects on romantic relationships.

The analyses revealed that Media Aware resulted in several immediate effects on descriptive norms about sexual activity, thus providing evidence that a media literacy education intervention can positively impact the mechanisms by which media exposure is thought to influence adolescent sexual behavior (see the Integrative Model of Behavioral Prediction; Bleakley et al., 2011). Despite the fact that less than half of teens have had sex (Kann et al., 2018), the frequency of teen sex (and risky sexual behaviors) in media messages presents a different picture. The program includes educational content that redresses the realism of media messages that depict teen sex and risky sexual behaviors as normative, and provides medicallyaccurate content about the actual frequency of these behaviors. Media Aware was found to reduce girls' descriptive normative beliefs about the percentage of teens who are sexually active and boys' and girls' descriptive normative beliefs about the percentage of teens who engage in risky sexual behaviors (i.e., having sex with someone who is much older; engaging in sex outside a relationship). 
Descriptive norms regarding peer sexual activity are associated with sexual intentions, and intentions are a stable predictor of adolescent sexual behaviors (Buhi \& Goodson, 2007). Given that there are not consistent gender differences in the percentage of high school students who are sexually active or engaging in risky sexual behaviors, it is not clear why this gender difference emerged. Future research is needed to better understand gender differences in both normative beliefs about teen sexual activity and the effectiveness of sexual health education.

Media Aware is one of relatively few sexual health programs to also address relationship health promotion and sexual violence prevention. The evaluation revealed that the program reduced boys' acceptance of dating violence. This is an important finding given that teen dating violence is a significant issue in the U.S., and the acceptance of dating violence is related to teen dating violence behaviors (Reyes, Foshee, Niolon, Reidy, \& Hall, 2016). Encouragingly, all beliefs related to dating violence and sexual violence measured in this study (i.e., acceptance of dating violence, stereotypical gender roles, and rape myths) were quite low to begin with as participants were unlikely to endorse these harmful beliefs. In this study, the program did not significantly impact bystander efficacy or bystander intentions as was demonstrated in the feasibility study. This may be a result of the timeframes when the two studies were conducted, where in the past high schools may have been less likely to have offered bystander intervention training to students or the students in the one high school in the feasibility study might overall have been less familiar with bystander intervention and therefore, more likely to benefit from the program. Whatever the case, the results from this RCT are still encouraging and argue for continuing to address the beliefs that contribute to violence, which are normalized across media formats, such as victim blaming, loss of agency, and not intervening in potential situations of potential sexual assault or intimate partner violence.

A key focus in Media Aware is building motivation and skills for sexual health communication. Communication about sexual health topics promotes safer-sex decisionmaking, whether it be with parents (Widman, ChoukasBradley, Noar, Nesi, \& Garrett, 2016) or partners (Noar, Carlyle, \& Cole, 2006). Likewise, adolescents must have some capacity to discuss sexual health topics with medical professionals to access STI testing and certain contraceptives. The program resulted in an immediate impact of girls' frequency of sexual health communication with a parent. It's not readily clear why the program enhanced girls' communication behaviors more; however, future research should collect more data regarding which parent was communicated with and the topics discussed between adolescents and their parents. There is research to suggest that parent-gender differences in parent-adolescent sexual health communication exist (Scull, Carl, Keefe, \& Malik, 2021), and mothers are often the primary parent engaging in sexual health communication with their child. Girls may have felt more comfortable engaging their parent (usually the mother) in sexual health communication than sons. In addition, boys' communication may have been suppressed by gender role stereotypes that suggest that males do not engage in sensitive conversations such as those about sexual health. Girls may also have been motivated to engage in sexual health conversations due, in part, to gender role stereotypes that suggest that sexual health is primarily a female responsibility. Future research is needed to better understand gender differences in adolescent sexual health communication, and how to encourage effective sexual health communication, especially between parents and sons.

This research has several strengths, which contribute to its scientific rigor. First, the generalizability of the results was enhanced by the participation of adolescents from high schools in different geographic locations from across the United States. In addition, the study included a range of high schools, including large public high schools, smaller charter schools, and alternative schools, and the resulting adolescent sample was diverse in terms of racial and ethnic identity. Second, intent-to-treat analyses provide a more realistic examination of the program whereby not all participants are compliant (e.g., completed the program) and thus avoids overestimates of program effects. Third, the web-based program was housed in an LMS that captured data on adolescents' use of the program and their quiz scores, which provided a useful and reliable measure of program implementation and satisfaction. Most adolescents completed the program and reported high levels of satisfaction with the experience, especially related to the webbased format of the program. Fourth, the results revealed that many of the findings found in the preliminary study of the program were replicated, thus providing converging evidence for the hypothesized impacts that the program is having on adolescents' media, sexual health, and communication outcomes.

The limitations of this research are also important to consider. First, intent-to-treat analyses, while having strengths, can also underestimate program effects; thus, this line of research could benefit from analyses that model compliance to better pinpoint program effects (e.g., complier average causal effects analyses). Second, the original study design included three timepoints in order to study behavioral change and mediation; however, there were challenges associated with the COVID-19 pandemic (e.g., procedural changes for data collection; significant environmental changes that impact sexual behaviors). Therefore, the final follow-up sample size impacted the study power to detect follow-up differences, examine behavioral change, and conduct mediation analyses. Additional research 
involving longer-term follow-up periods and mediation analyses are crucial to deepening our understanding of the mechanisms whereby media literacy education-based programs promote a change in health behaviors. Third, although participants were able to self-identify their gender identity, the moderator analyses focused on the impact of the program for adolescents who identified as either boy or girl (i.e., gender binary).

\section{Conclusion}

Media may function as sex educators for many adolescents; unfortunately, media messages often glamorize risky sexual behaviors and unhealthy relationships and neglect sexual health behaviors and communication. The present study extends preliminary research on Media Aware, a high school comprehensive sexual health program that uses a media literacy education approach by evaluating it with a diverse sample of adolescents from a much larger number of high schools across the United States. An attempt was made to examine if program effects persist over time, but this goal was impacted by the COVID-19 pandemic. Despite this, the study revealed several immediate impacts of the program for students, including reductions in the perceived realism of media messages, improvements in media message deconstruction skills, increases in sexual health knowledge, and reductions in normative beliefs about the frequency of risky teen sexual activity. Gender-specific effects of the program included a reduction in boys' acceptance of dating violence and girls' normative beliefs about the frequency of teen sexual activity and an increase in the frequency that girls communicate about sexual health with a parent. Finally, adolescents reported favorable experiences completing Media Aware. The findings in both the preliminary and current study of Media Aware related to descriptive normative beliefs points to a potential mediating mechanism of the program that warrants additional research. Media are central in the lives of adolescents; this study suggests that media literacy education may redress the potentially negative impact of unhealthy sexual media messages by building critical media message processing skills in adolescence.

Acknowledgements We wish to thank the teachers and students who helped with this study.

Authors' Contributions T.M.S. and C.V.D. designed the trial, developed the intervention program and resources, wrote the analytic plan, supervised the study implementation, interpreted the data, and drafted the manuscript; J.G.G. participated in the design and coordination of the study, performed the measurement, and helped to draft the manuscript; L.C.R. participated in the coordination of the study, performed the measurement, and helped to draft the manuscript; K.N.S. aided with analyses and interpretation and helped to draft the manuscript. All authors read and approved the final manuscript.
Funding This work was supported by the Eunice Kennedy Shriver National Institute of Child Health and Human Development of the National Institutes of Health under award number R44HD088254 to the first author. Research reported in this paper is solely the responsibility of the authors and does not necessarily represent the official views of the National Institutes of Health.

Data Sharing Declaration The datasets generated and/or analyzed during the current study are not publicly available but are available from the corresponding author on reasonable request.

Preregistration The RCT described in this manuscript was preregistered in www.clinicaltrials.gov (NCT04035694), and the protocol for the study was previously published in Trials (Scull, Malik, Morrison, \& Keefe, 2020).

\section{Compliance with Ethical Standards}

Conflict of Interest The authors are employed by a small business. The business has a financial interest in the copyright and sale of the Media Aware program for research and clinical purposes.

Ethical Approval All procedures performed in the current study were in accordance with the ethical standards of the institutional and/ or national research committee and with the 1964 Helsinki declaration and its later amendments or comparable ethical standards.

Informed Consent Informed consent was obtained for all participants in the study.

Publisher's note Springer Nature remains neutral with regard to jurisdictional claims in published maps and institutional affiliations.

\section{References}

Ajzen, I. (1991). The theory of planned behavior. Organizational Behavior and Human Decision Processes, 50, 179-211.

Ajzen, I., \& Fishbein, M. (1975). Belief, attitude, intention, and behavior: an introduction to theory and research. Reading, MA: Addison-Wesley.

Alexopoulos, C., \& Taylor, L. D. (2020). Risky business: sexual risk and responsibility messages in teen sex romps. Sexuality \& Culture, 24 (6), 2161-2182. https://doi.org/10.1007/s12119-020-09742-4.

Aufderheide, P. (1993). Media literacy. a report of the national leadership conference on media literacy.

Austin, E. W., \& Johnson, K. K. (1997). Effects of general and alcohol-specific media literacy training on children's decision making about alcohol. Journal of Health Communication, 2(1), 17-42. https://doi.org/10.1080/108107397127897.

Austin, E. W., \& Johnson, K. K. (1997a). Effects of general and alcohol-specific media literacy training on children's decision making about alcohol. Journal of Health Communication, 2, $17-42$.

Banyard, V. L., Plante, E. G., \& Moynihan, M. M. (2005). Rape prevention through bystander education: bringing a broader community perspective to sexual violence prevention. U.S. Department of Justice.

Bleakley, A., Hennessy, M., Fishbein, M., \& Jordan, A. (2011). Using the Integrative Model to explain how exposure to sexual media content influences adolescent sexual behavior. Health Education \& Behavior, 38(5), 530-540. https://doi. org/10.1177/1090198110385775. 
Borawski, E. A., Tufts, K. A., Trapl, E. S., Hayman, L. L., Yoder, L. D. \& Lovegreen, L. D. (2015). Effectiveness of health education teachers and school nurses teaching sexually transmitted infections/ human immunodeficiency virus prevention knowledge and skills in high school. The Journal of School Health, 85(3), 189-196.

Bridges, A. J., Wosnitzer, R., Scharrer, E., Sun, C., \& Liberman, R. (2010). Aggression and sexual behavior in best-selling pornography videos: a content analysis update. Violence Against Women, 16(10), 1065-1085. https://doi.org/10.1177/1077801210382866.

Brown, J. D., Halpern, C. T., \& L'Engle, K. L. (2005). Mass media as a sexual super peer for early maturing girls. Journal of Adolescent Health, 36(5), 420-427. https://doi.org/10.1016/j.jadohealth. 2004.06.003.

Buhi, E. R., \& Goodson, P. (2007). Predictors of adolescent sexual behavior and intention: a theory-guided systematic review. Journal of Adolescent Health, 40(1), 4-21. https://doi.org/10. 1016/j.jadohealth.2006.09.027.

Chaiken, S., Giner-Sorolla, R., \& Chen, S. (1996). Beyond accuracy: defense and impression motives in heuristic and systematic information processing. In P. M. Gollwitzer (Ed.), Psychology of action: linking cognition and motivation to behavior (pp. 553-578). New York, NY: Guilford Press.

Coyne, S. M., Ward, L. M., Kroff, S. L., Davis, E. J., Holmgren, H. G., Jensen, A. C.,... Essig, L. W. (2019). Contributions of mainstream sexual media exposure to sexual attitudes, perceived peer norms, and sexual behavior: a meta-analysis. Journal of Adolescent Health. https://doi.org/10.1016/j.jadohealth.2018.11.016

Crosby, R. A., Hanson, A., \& Rager, K. (2009). The protective value of parental sex education: a clinic-based exploratory study of adolescent females. Journal of Pediatric Adolescent Gynecology, 22(3), 189-192. https://doi.org/10.1016/j.jpag.2008.08.006.

Dillman Carpentier, F. R., Stevens, E. M., Wu, L., \& Seely, N. (2017). Sex, love, and risk-n-responsibility: a content analysis of entertainment television. Mass Communication and Society, 20(5), 686-709. https://doi.org/10.1080/15205436.2017.1298807.

Finkelhor, D., Shattuck, A., Turner, H. A., \& Hamby, S. L. (2014). The lifetime prevalence of child sexual abuse and sexual assault assessed in late adolescence. Journal of Adolescent Health, 55(3), 329-333. https://doi.org/10.1016/j.jadohealth.2013.12.026.

Foshee, V. A., Bauman, K. E., Ennett, S. T., Suchindran, C., Benefield, T., \& Linder, G. F. (2005). Assessing the effects of the dating violence prevention program "Safe Dates" using random coefficient regression modeling. Prevention Science, 6(3), 245-258. https://doi.org/10.1007/s11121-005-0007-0.

Gerrard, M., Gibbons, F. X., Houlihan, A. E., Stock, M. L., \& Pomery, E. A. (2008). A dual-process approach to health risk decision making: the prototype willingness model. Developmental Review, 28(1), 29-61. https://doi.org/10.1016/j.dr.2007.10.001.

Gibbons, F. X., Gerrard, M., Blanton, H., \& Russell, D. W. (1998). Reasoned action and social reaction: Willingness and intention as independent predictors of health risk. Journal of Personality and Social Psychology, 74(5), 1164-1180.

Goldfarb, E. S., \& Lieberman, L. D. (2021). Three decades of research: the case for comprehensive sex education. Journal of Adolescent Health, 68(1), 13-27. https://doi.org/10.1016/j.jadohealth.2020.07.036.

Grudzen, C. R., Elliott, M. N., Kerndt, P. R., Schuster, M. A., Brook, R. H., \& Gelberg, L. (2009). Condom use and high-risk sexual acts in adult films: a comparison of heterosexual and homosexual films. American Journal of Public Health, 99(Suppl 1), S152-S156. https://doi.org/10.2105/AJPH.2007.127035.

Haberland, N., \& Rogow, D. (2015). Sexuality education: emerging trends in evidence and practice. Journal of Adolescent Health, 56 , S15-S21.

Jemmott, L. S., \& Jemmott, J. B. (1991). Applying the theory of reasoned action to AIDS risk behavior: condom use among black women. Nursing Research, 40, 228-234.
Johnston, J. B., \& Dill-Shackleford, K. (2020). Rape myth acceptance. In J. Bulck (Ed.), The international encyclopedia of media psychology (pp. 1-7).

Jozkowski, K. N., Marcantonio, T. L., Rhoads, K. E., Canan, S., Hunt, M. E., \& Willis, M. (2019). A content analysis of sexual consent and refusal communication in mainstream films. Journal of Sex Research, 1-12. https://doi.org/10.1080/00224499.2019.1595503

Kann, L., McManus, T., Harris, W. A., Shanklin, S. L., Flint, K. H., Queen, B.,... Ethier, K. A. (2018). Youth risk behavior surveillance-United States, 2017.

Kim, J. L., \& Wells, B. E. (2017). Assessing alcohol and sexual content on reality dating programs. Psychology of Popular Media Culture, 6(3), 237-254. https://doi.org/10.1037/ppm0000098.

Kirby, D., \& Laris, B. A. (2009). Effective curriculum-based sex and STD/HIV education programs for adolescents. Child Development Perspectives, 3(1), 21-29.

L'Engle, K. L., Brown, J. D., \& Kenneavy, K. (2006). The mass media are an important context for adolescents' sexual behavior. Journal of Adolescent Health, 38(3), 186-192. https://doi.org/10. 1016/j.jadohealth.2005.03.020.

Liao, L.-L., Chang, L.-C., Lee, C.-K., \& Tsai, S.-Y. (2020). The effects of a television drama-based media literacy initiative on Taiwanese adolescents' gender role attitudes. Sex Roles, 82(3-4), 219-231. https://doi.org/10.1007/s11199-019-01049-5.

MacKenzie, S. B., Lutz, R. J., \& Belch, G. E. (1986). The role of attitude toward the ad as a mediator of advertising effectiveness: a test of competing explanations. Journal of Marketing Research, 23(2), 130-143. https://doi.org/10.2307/3151660.

McMahon, S., \& Farmer, L. (2011). An updated measure for assessing subtle rape myths. National Association of Social Workers, 35(2), $71-81$.

Moyer-Gusé, E., \& Nabi, R. L. (2010). Explaining the effects of narrative in an entertainment television program: overcoming resistance to persuasion. Human Communication Research, 36 (1), 26-52. https://doi.org/10.1111/j.1468-2958.2009.01367.x.

Noar, S. M., Carlyle, K., \& Cole, C. (2006). Why communication is crucial: meta-analysis of the relationship between safer sexual communication and condom use. Journal of Health Communication, 11 (4), 365-390. https://doi.org/10.1080/10810730600671862.

O’Hara, R. E., Gibbons, F. X., Gerrard, M., Li, Z., \& Sargent, J. D. (2012). Greater exposure to sexual content in popular movies predicts earlier sexual debut and increased sexual risk taking. Psychological Science, 23(9), 984-993. https://doi.org/10.1177/ 0956797611435529.

Peter, J., \& Valkenburg, P. M. (2016). Adolescents and pornography: a review of 20 years of research. Journal of Sex Research, 53(4-5), 509-531. https://doi.org/10.1080/00224499.2016.1143441.

Petty, R. E., \& Cacioppo, J. T. (1986). The elaboration likelihood model of persuasion. In Springer Series in Social Psychology (Ed.), Communication and persuasion. New York, NY: Springer.

Pew Research Center. (2018). Teens, Social Media \& Technology 2018.

Raudenbush, S. W., Spybrook, J., Bloom, H., Congdon, R., Hill, C., \& Martinez, A. (2011). Optimal design software for multi-level and longitudinal research (Version 3.01) \{Software].

Reyes, H. L. M., Foshee, V. A., Niolon, P. H., Reidy, D. E., \& Hall, J. E. (2016). Gender role attitudes and male adolescent dating violence perpetration: normative beliefs as moderators. Journal of Youth and Adolescence, 45(2), 350-360. https://doi.org/10.1007/ s10964-015-0278-0.

Rideout, V., \& Robb, M. B. (2019). The common sense census: media use by tweens and teens.

Rivis, A., \& Sheeran, P. (2003). Descriptive norms as an additional predictor in the theory of planned behaviour: A meta-analysis. Current Psychology, 22(3), 218-233. https://doi.org/10.1007/ s12144-003-1018-2. 
Rodenhizer, K. A. E., \& Edwards, K. M. (2019). The Impacts of sexual media exposure on adolescent and emerging adults' dating and sexual violence attitudes and behaviors: a critical review of the literature. Trauma, Violence, \& Abuse, 20(4), 439-452. https://doi.org/10.1177/1524838017717745.

Rodgers, K. B., Hust, S. J. T., Willoughby, J. F., Wheeler, J., \& Li, J. (2019). Adolescents' sex-related alcohol expectancies and alcohol advertisements in magazines: the role of wishful identification, realism, and beliefs about women's enjoyment of sexualization. Journal of Health Communication, 24(4), 395-404. https://doi.org/10.1080/10810730.2019.1630523.

Rodgers, R. F., McLean, S. A., \& Paxton, S. J. (2018). When seeing is not believing: an examination of the mechanisms accounting for the protective effect of media literacy on body image. Sex Roles, 81(1-2), 87-96. https://doi.org/10.1007/s11199-018-0973-x.

Rothman, E. F., Adhia, A., Christensen, T. T., Paruk, J., Alder, J., \& Daley, N. (2018). A pornography literacy class for youth: results of a feasibility and efficacy pilot study. American Journal of Sexuality Education, 13(1), 1-17. https://doi.org/10.1080/ 15546128.2018.1437100.

Rubin, D. B. (1987). Multiple imputation for nonresponse in surveys. New York: Wiley.

Scull, T. M., Carl, A. E., Keefe, E. M., \& Malik, C. V. (2021). Exploring parent-gender differences in parent and adolescent reports of the frequency, quality, and content of their sexual health communication. Journal of Sex Research, 1-13. https:// doi.org/10.1080/00224499.2021.1936439

Scull, T. M., Kupersmidt, J. B., Malik, C. V., \& Keefe, E. M. (2018). Examining the efficacy of an mHealth media literacy education program for sexual health promotion in older adolescents attending community college. Journal of American College Health, 66(3), 165-177.

Scull, T. M., Kupersmidt, J. B., Malik, C. V., \& Morgan-Lopez, A. A. (2018). Using media literacy education for adolescent sexual health promotion in middle school: randomized control trial of Media Aware. Journal of Health Communication, 23 (12), 1051.

Scull, T. M., Malik, C. V., Keefe, E. M., \& Schoemann, A. (2019). Evaluating the short-term impact of Media Aware Parent, a webbased program for parents with the goal of adolescent sexual health promotion. Journal of Youth Adolescence, 48(9), 1686-1706. https://doi.org/10.1007/s10964-019-01077-0.

Scull, T. M., Malik, C. V., \& Kupersmidt, J. B. (2014). A media literacy education approach to teaching adolescents comprehensive sexual health education. Journal of Media Literacy Education, 6(1), 1-14.

Scull, T. M., Malik, C. V., Morrison, A., \& Keefe, E. M. (2020). Study protocol for a randomized controlled trial to evaluate a web-based comprehensive sexual health and media literacy education program for high school students. Trials, 21(1), $50 \mathrm{https}: / /$ doi.org/10. 1186/s13063-019-3992-1.

Scull, T. M., Malik, C. V., Morrison, A., \& Keefe, E. M. (2021). Promoting sexual health in high school: a feasibility study of a webbased media literacy education program. Journal of Health Communication, 1. https://doi.org/10.1080/10810730.2021.1893868

Sedgh, G., Finer, L. B., Bankole, A., Eilers, M. A., \& Singh, S. (2015). Adolescent pregnancy, birth, and abortion rates across countries: levels and recent trends. Journal of Adolescent Health, 56(2), 223-230. https://doi.org/10.1016/j.jadohealth.2014.09.007.

Shiv, B., Britton, J. A. E., \& Payne, J. W. (2004). Does elaboration increase or decrease the effectiveness of negatively versus positively framed messages? Journal of Consumer Research, 31(1), 199-208.

Smiler, A. P., Shewmaker, J. W., \& Hearon, B. (2017). From "I Want To Hold Your Hand" to "Promiscuous": sexual stereotypes in popular music lyrics, 1960-2008. Sexuality \& Culture, 21(4), 1083-1105. https://doi.org/10.1007/s12119-017-9437-7.

Smith, L. W., Liu, B., Degenhardt, L., Richters, J., Patton, G., Wand, H., \& Guy, R. (2016). Is sexual content in new media linked to sexual risk behaviour in young people? A systematic review and metaanalysis. Sexual Health, 13(6), 501-515. https://doi.org/10.1071/ SH16037.

Soet, J. E., Dudley, W. N., \& Dilorio, C. (1999). The effects of ethnicity and perceived power on women's sexual behavior. Psychology of Women Quarterly, 23, 707-723.

Vahedi, Z., Sibalis, A., \& Sutherland, J. E. (2018). Are media literacy interventions effective at changing attitudes and intentions towards risky health behaviors in adolescents? A meta-analytic review. Journal of Adolescence, 67, 140-152. https://doi.org/10. 1016/j.adolescence.2018.06.007.

van Oosten, J. M. F., Peter, J., \& Vandenbosch, L. (2017). Adolescents' sexual media use and willingness to engage in casual sex: Differential relations and underlying processes. Human Communication Research, 43(1), 127-147. https://doi.org/10.1111/ hcre. 12098.

Ward, L. M., Erickson, S. E., Lippman, J., \& Giaccardi, S. (2016). Sexual media content and effects. In J. F. Nussbaum (Ed.), Oxford research encyclopedia of communication (Vol. 1): Oxford University Press.

Widman, L., Choukas-Bradley, S., Noar, S. M., Nesi, J., \& Garrett, K. (2016). Parent-adolescent sexual communication and adolescent safer sex behavior: a meta-analysis. JAMA Pediatrics, 170(1), 52-61. https://doi.org/10.1001/jamapediatrics.2015.2731.

Widman, L., Noar, S. M., Choukas-Bradley, S., \& Francis, D. B. (2014). Adolescent sexual health communication and condom use: a meta-analysis. Health Psychology, 33(10), 1113-1124. https://doi.org/10.1037/hea0000112.

Tracy M. Scull is a Senior Research Scientist at innovation Research $\&$ Training. Her major research interests include substance abuse prevention, sexual health promotion, media effects, media literacy education, and development from childhood through young adulthood.

Christina V. Dodson is a Research Scientist at innovation Research $\&$ Training. Her research focuses on the influence of media on health behaviors and the role of critical media analysis in the processing of media messages.

Jacob G. Geller B.S., is a Research Assistant at innovation Research \& Training. He specializes in media literacy education and research communication.

Liz C. Reeder B.S., is a Research Assistant at innovation Research \& Training. Her research interests include sexual health promotion, sex and sexuality, mindfulness, and adolescent development and mental health.

Kathryn N. Stump is a Research Scientist at innovation Research \& Training. Her research interests include youth mentoring, substance abuse and delinquency prevention, peer relations, and program evaluation. 